\section{(- OPEN ACCESS}

\title{
Fever of unknown origin: a challenging case
}

\author{
Sarah Vella, ${ }_{1}^{1}$ Bernard Coleiro, ${ }^{2}$ Charles Mallia Azzopardi ${ }^{3}$
}

${ }^{1}$ Medicine, Mater Dei Hospital, Msida, Malta

${ }^{2}$ Rheumatology, Mater Dei Hospital, Msida, Malta

${ }^{3}$ Infectious Diseases, Mater Dei Hospital, Msida, Malta

\section{Correspondence to}

Dr Sarah Vella,

smv2610@gmail.com

Accepted 15 May 2018

Check for updates

To cite: Vella S, Coleiro B, Mallia Azzopardi C. BMJ Case Rep Published Online First: [please include Day Month Year]. doi:10.1136/bcr-2017224031

\section{SUMMARY}

We report a case of Cogan's syndrome presenting as fever of unknown origin in a 31-year-old woman who was admitted to the hospital with a 7-week history of fever, night sweats and other constitutional symptoms. The diagnosis remained elusive despite numerous investigations, and the patient subsequently developed rash, episcleritis, dizziness and sensorineural hearing loss. While initially thought to be a postinflammatory response to a previous infection, confirmation of the rash as a vasculitis together with the audiovestibular and ocular involvement led to a clinical diagnosis of Cogan's syndrome. This was further corroborated by resolution of her symptoms once immunosuppressive therapy was instituted. Early recognition of Cogan's syndrome is crucial to reducing the risk of serious complications through the timely initiation of treatment.

\section{BACKGROUND}

Fever of unknown origin (FUO) is defined as a body temperature of $\geq 38.3^{\circ} \mathrm{C}$ on at least two occasions and a duration of illness of $\geq 3$ weeks or having multiple febrile episodes over this time period. There must be no obvious diagnosis despite complete history taking and examination, as well as appropriate investigation that should include routine blood tests, inflammatory markers, autoimmune screen, cultures, chest radiography and abdominal imaging. ${ }^{1}$ Investigation includes identifying any potential diagnostic clues (PDCs) that can help reveal the underlying cause of the fever; however, up to $50 \%$ of cases of FUO remain undiagnosed. ${ }^{2}$

The case described here is of a rare condition presenting with FUO that proved to be a diagnostic challenge. Despite its rarity, accurate documentation of cases of Cogan's syndrome remains crucial to improving our awareness and understanding of this condition. This could in turn bring about the development of more effective treatment as well as reduce patient morbidity by leading to earlier diagnosis. Our case also stresses how laboratory and radiological investigations should not serve as an alternative to thorough clinical assessments since early recognition of PDCs and symptom complexes is more likely to help clinch a diagnosis than investigations which can at times return non-specific results.

\section{CASE PRESENTATION}

A 31-year-old Caucasian female smoker was admitted to the hospital with a 7-week history of fever associated with night sweats, joint pains, myalgia and nasal congestion.
She had already been hospitalised 3 weeks prior to this admission, at which time she was treated with empirical antibiotics for a presumed community-acquired pneumonia as CT scan of the thorax/ abdomen/pelvis had revealed subsegmental patchy consolidation of the left lower lobe of the lung.

The patient was readmitted as she continued to have fever despite completing the course of antibiotics prescribed. The history was reviewed in detail with special attention being given to social, sexual and travel history. She had been working in a glass factory for a number of years and had not left the country in recent months. There was no family or sexual history of note and she had no contact with animals apart from her healthy pet dog. The fever had been going on for a total of 7 weeks, and she had also been experiencing drenching night sweats, joint pains and myalgias, with recent development of dizziness and hearing impairment. She denied any gastrointestinal symptoms. On examination she was noted to have conjunctival injection secondary to episcleritis and a non-pruritic maculopapular rash over the upper limbs (figure 1), as well as nodular, tender lesions over her lower limbs. There were no orogenital ulcerations or joint swellings and she was neurologically intact.

\section{INVESTIGATIONS}

Routine blood investigations were unremarkable apart from a normocytic normochromic anaemia with a high reticulocyte count, a neutrophilic leucocytosis and raised inflammatory markers (figure 2). Blood and urine cultures, as well as an extensive viral screen which included serology for Epstein-Barr virus, Cytomegalovirus and HIV, were negative.

Systemic vasculitis such as granulomatosis with polyangiitis was excluded as repeat autoimmune screens, which included ANA (antinuclear antibody), ANCA (anti-neutrophil cytoplasmic antibody), ASMA (anti-smooth muscle antibody), rheumatoid factor, anti-CCP (anti-cyclic citrullinated peptide) and complement levels, were negative. Repeat imaging of the chest showed complete resolution of the previous pulmonary consolidation following antibiotic treatment. This, together with the absence of lymphadenopathy, a normal serum calcium and negative serum ACE, excluded a diagnosis of sarcoidosis, and no further pulmonary investigations were considered.

Serological tests for Mycoplasma, Bartonella, Coxiella, Brucella, Borrelia burgdorferi and Chlamydia, as well as for other infective conditions including syphilis and leishmania, were negative. Tests for antistreptolysin $\mathrm{O}$ titre, cryoglobulins and 


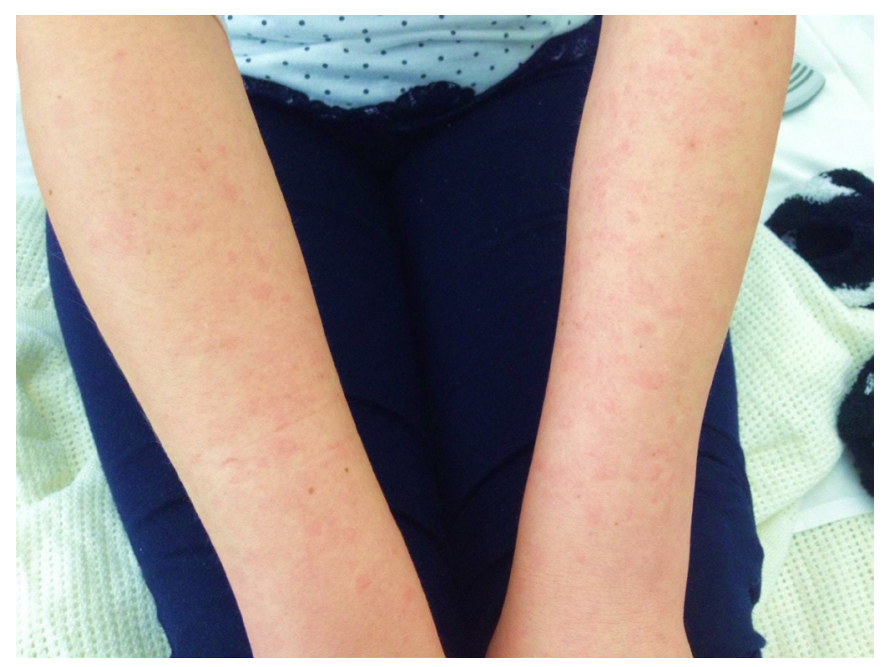

Figure 1 Upper limb maculopapular rash.

cold agglutinins were also performed in order to rule out an underlying infectious cause.

An MRI of the patient's brain was performed in view of her audiovestibular symptoms; however, this only revealed longstanding mastoid effusions.

\section{DIFFERENTIAL DIAGNOSIS}

The causes of FUO can be grouped as infectious, non-infectious inflammatory disorders, malignancy, and miscellaneous which includes conditions such as thyrotoxicosis. All of the possible causes in these categories which could potentially explain the clinical picture in this case, including rare ones, were considered and excluded following intensive investigation. One such example was syphilis, which can present with similar ocular manifestations. The absence of orogenital ulceration made Behçet's unlikely, and inflammatory bowel disease was excluded on the basis of the patient not having gastrointestinal symptoms and a negative faecal calprotectin.

A provisional diagnosis of postinflammatory reaction to a previous infection complicated by low-grade haemolysis with possible myringitis was made, with both of these being recognised complications of Mycoplasma pneumoniae infection. The patient was prescribed doxycycline together with a non-steroidal anti-inflammatory agent for fever control and prochlorperazine to help relieve the dizziness.

A week after being discharged on the above treatment, her symptoms had not improved. In view of her worsening hearing impairment, audiometry was performed and this confirmed bilateral sensorineural hearing loss. Biopsies from the lower limb rash showed a perivascular lymphocytic infiltrate typical of small-sized and medium-sized vessel vasculitis.

On considering all of the patient's symptoms and their evolution, a tentative diagnosis of Cogan's syndrome was entertained. She had gone on to develop all the classical features of the condition, that is, audiovestibular and ocular involvement together with vasculitis.

\section{TREATMENT}

After being diagnosed with Cogan's syndrome, the patient was started on oral prednisolone therapy with subsequent addition of methotrexate and folic acid.

\section{OUTCOME AND FOLLOW-UP}

Within a week of starting systemic steroids, our patient became afebrile with significant improvements in both her skin rash and hearing. She was advised to gradually tail off her steroids but returned soon after in view of flare-up of all her prior symptoms, including low-grade fever, skin lesions, hearing impairment and conjunctival suffusion. It was also noted that her inflammatory markers had started to increase again (figure 2).

The patient was reviewed by a rheumatologist who agreed with the diagnosis and started her on methotrexate and folic acid with more gradual tailing down of steroids. The patient has

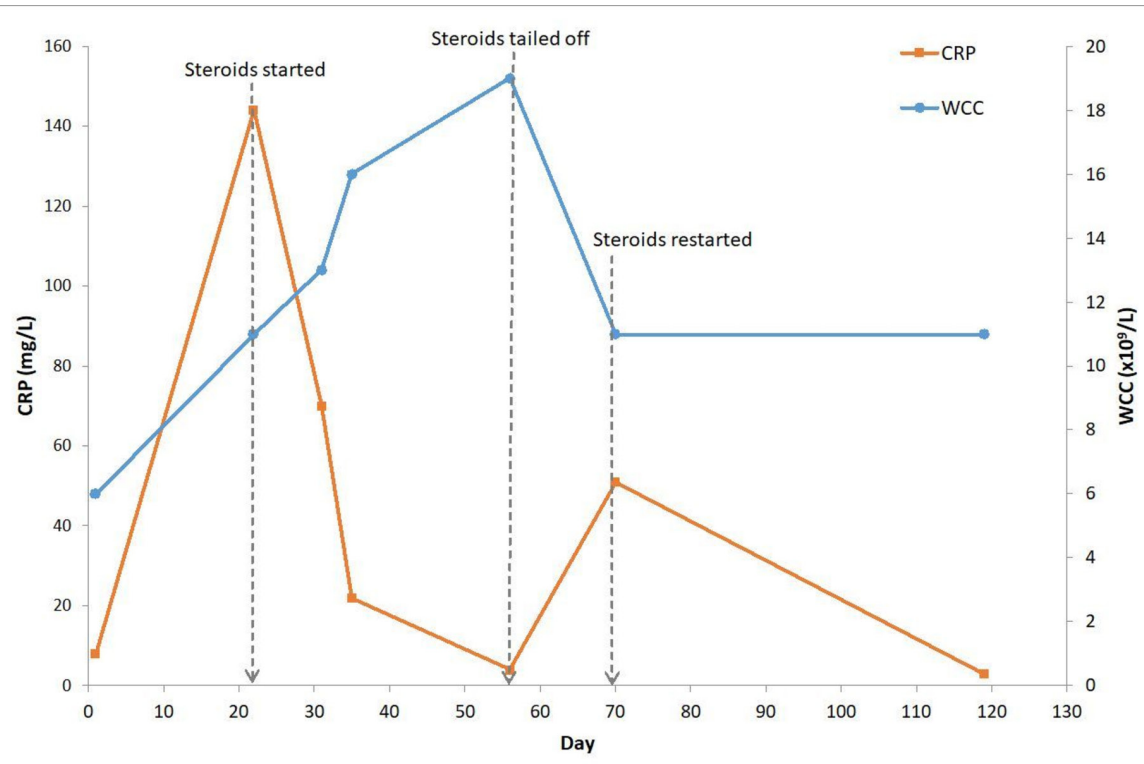

Figure 2 Graph showing trend in the patient's white cell count (WCC) and C reactive protein (CRP). High CRP level improved once steroid therapy was initiated; however, a second rise was noted on tailing down of the dose. The persistently elevated WCC can be attributed to that same steroid therapy. 
since remained stable on methotrexate, folic acid and low-dose steroid therapy.

\section{DISCUSSION}

Cogan's syndrome is a rare immune-mediated vasculitis that mostly affects young adults with no known gender predominance. Diagnosis is based on the characteristic involvement of the eyes and inner ear. The condition was first described in the 1940s, and since then about 250 cases have been reported. ${ }^{3}$ There may also be an infective aspect to its aetiology with a viral prodrome to the illness being common, as well as the fact that Chlamydia species have been isolated in a number of patients. ${ }^{4}$ Cigarette smoking has been identified as a possible trigger, and a number of patients were also known sufferers of inflammatory bowel disease. ${ }^{5}{ }^{6}$ The previous categorisation of Cogan's syndrome as either typical or atypical is no longer used as it did not carry any prognostic significance.

The two major characteristics of the condition are ocular inflammation, classically in the form of interstitial keratitis (IK), and audiovestibular dysfunction. IK is not essential for the diagnosis to be made as ocular inflammation may involve other parts of the eye, resulting in conjunctivitis or episcleritis as described in the case above. ${ }^{8}$ Vestibuloauditory dysfunction presents with vertigo, nausea, tinnitus and/or sensorineural hearing loss, which can become profound with recurrent or chronic disease.

In addition to the characteristic features, numerous systemic manifestations can occur, most commonly affecting the cardiovascular, neurological and gastrointestinal systems, with aortitis being known to affect about $10 \%$ of patients. ${ }^{9}$ Less than $5 \%$ of patients initially present with solely systemic upset; however, in these cases diagnosis can only be made once eye or ear disease develops. Non-specific symptoms such as fever, arthralgia, myalgia and weight loss have also been documented to occur.

A similar case to ours of a 31-year-old woman was described in 2009 by Migliori et al. ${ }^{10}$ She had initially presented with vertigo, hearing loss and tinnitus, as well as conjunctivitis. A working diagnosis of herpes zoster infection was made, and the patient was treated with steroids and valacyclovir. It was only on her second admission 10 days later that Cogan's syndrome was suspected as she had developed more severe hearing loss that was confirmed to be sensorineural. Other cases have been described all showing the diagnostic challenge posed by this condition, including that reported by Al-Shagahin and Al-Hamaidah $^{3}$ of a Jordanian patient who was only diagnosed after 6 months of intensive investigation.

There is no specific diagnostic test for Cogan's syndrome so one must have a high index of suspicion as even cranial imaging is often normal, with signals of active disease being picked up in the inner ear of only a small proportion of patients. ${ }^{3}$ Being a clinical diagnosis, it is essential that other possible differentials are excluded before making a diagnosis of Cogan's syndrome. Other conditions that can manifest with simultaneous eye and ear disease are sarcoidosis, Whipple's disease, rheumatoid arthritis, granulomatosis with polyangiitis, Behçet's syndrome, inflammatory bowel disease, syphilis and lympho/myeloproliferative disease of the central nervous system. The diagnosis in our patient was narrowed down to Cogan's syndrome after such other conditions were excluded by way of repeat history taking and examination, as well as extensive investigation.

Patients with sudden or rapidly progressive hearing loss should be closely followed up as this could represent the initial manifestation of Cogan's syndrome. Based on their case Migliori et al ${ }^{10}$ advised monthly vestibuloauditory assessment for the first 3 months, then 3 monthly for the first 2 years, every 6 months in the third year and yearly thereafter.

Early treatment is important in order to limit progression of hearing loss. First-line treatment is with corticosteroids. Topical agents can be used for mild eye disease, which may even resolve without any treatment. Systemic steroids are needed for more extensive ocular involvement, as well as for inner ear disease and systemic vasculitis. If hearing improves steroids can be tapered over 2-6 months, but more prolonged treatment requires the addition of steroid-sparing agents such as methotrexate. ${ }^{3}$ Cochlear implants have been successful in cases where pharmacological therapy has failed. Life expectancy is normal in the absence of systemic involvement. ${ }^{11}$

\section{Learning points}

- History and examination should be repeated throughout the course of the investigation of a fever of unknown origin as these are crucial to identifying potential diagnostic clues, particularly for conditions for which no specific confirmatory test exists.

- Longitudinal patient follow-up is essential in making a diagnosis as pathogneumonic features may not all be present at the outset, but tend to develop over a period of time.

- The differential diagnosis of a patient presenting with simultaneous eye and inner ear disease includes sarcoidosis, Whipple's disease, rheumatoid arthritis, granulomatosis with polyangiitis, Behçet's syndrome, inflammatory bowel disease, syphilis, lympho/myeloproliferative disease of the central nervous system and Cogan's syndrome.

- Cogan's syndrome is a challenging diagnosis that can easily be missed at first presentation.

- Delay in making the diagnosis should be avoided in view of the potentially disabling sequelae of this condition.

- Follow-up of any patient with sudden or rapidly progressive hearing loss should be guaranteed for at least 2 years as this could represent the initial manifestation of Cogan's syndrome.

Contributors SV was responsible for the literature review and manuscript preparation. BC and CMA contributed towards review and editing of the final manuscript.

Funding The authors have not declared a specific grant for this research from any funding agency in the public, commercial or not-for-profit sectors.

Competing interests None declared.

\section{Patient consent Obtained.}

Provenance and peer review Not commissioned; externally peer reviewed.

Open Access This is an Open Access article distributed in accordance with the Creative Commons Attribution Non Commercial (CC BY-NC 4.0) license, which permits others to distribute, remix, adapt, build upon this work non-commercially, and license their derivative works on different terms, provided the original work is properly cited and the use is non-commercial. See: http://creativecommons.org/ licenses/by-nc/4.0/

(c) BMJ Publishing Group Ltd (unless otherwise stated in the text of the article) 2018. All rights reserved. No commercial use is permitted unless otherwise expressly granted.

\section{REFERENCES}

1 Mulders-Manders C, Simon A, Bleeker-Rovers C. Fever of unknown origin. Clin Med 2015; 15:280-4. 
2 Bleeker-Rovers CP, Vos FJ, de Kleijn EM, et al. A prospective multicenter study on fever of unknown origin: the yield of a structured diagnostic protocol. Medicine 2007:86:26-38.

3 Al-Shagahin H, Al-Hamaidah AM. Cogan's Syndrome in a Jordanian patient: A case report. Alexandria Journal of Medicine 2014;50:377-80.

4 Belhoucha B, Belghmidi S, Benbaddan I, et al. Atypical Cogan's Syndrome: case report of an oculoaudiovestibular disease. American Journal of Medical Case Reports 2014:2:139-42.

5 Gluth MB, Baratz KH, Matteson EL, et al. Cogan syndrome: a retrospective review of 60 patients throughout a half century. Mayo Clin Proc 2006;81:483-8.

6 Vavricka SR, Greuter T, Scharl M, et al. Cogan's syndrome in patients with inflammatory bowel disease-a case series. J Crohns Colitis 2015;9:886-90.
7 Zulian F, Sari M, Zannin ME. Cogan's Syndrome. Parnham MJ, Compendium of inflammatory diseases. Basel, Switzerland: Springer, 2016:332-9.

8 Mazlumzadeh M, Matteson EL. Cogan's syndrome: an audiovestibular, ocular, and systemic autoimmune disease. Rheum Dis Clin North Am 2007;33:855-74. vii-viii.

9 Haynes BF, Kaiser-Kupfer MI, Mason P, et al. Cogan syndrome: studies in thirteen patients, long-term follow-up, and a review of the literature. Medicine 1980;59:426-41.

10 Migliori G, Battisti E, Pari M, et al. A shifty diagnosis: Cogan's syndrome. A case report and review of the literature. Acta Otorhinolaryngol Ital 2009;29:108-13.

11 Belghmidi S, Belhoucha B, Hajji I, et al. Typical and Atypical Cogan's Syndrome: 7 Cases and Review of the Literature. Neuro-Ophthalmology \& Visual Neuroscience 2014;1:8-12.

Copyright 2018 BMJ Publishing Group. All rights reserved. For permission to reuse any of this content visit http://group.bmj.com/group/rights-licensing/permissions.

BMJ Case Report Fellows may re-use this article for personal use and teaching without any further permission.

Become a Fellow of BMJ Case Reports today and you can:

- Submit as many cases as you like

- Enjoy fast sympathetic peer review and rapid publication of accepted articles

- Access all the published articles

Re-use any of the published material for personal use and teaching without further permission

For information on Institutional Fellowships contact consortiasales@bmjgroup.com

Visit casereports.bmj.com for more articles like this and to become a Fellow 